\title{
Control of Flexible Joint Robot Based on Motor State Feedback and Dynamic Surface Approach
}

\author{
Pengxiao Jia \\ School of Science, Beijing Forestry University, Beijing 100083, China \\ Correspondence should be addressed to Pengxiao Jia; jiapengxiao@126.com
}

Received 4 March 2019; Revised 14 May 2019; Accepted 23 May 2019; Published 1 July 2019

Academic Editor: Haiping Du

Copyright (C) 2019 Pengxiao Jia. This is an open access article distributed under the Creative Commons Attribution License, which permits unrestricted use, distribution, and reproduction in any medium, provided the original work is properly cited.

A controller based on dynamic surface control and observer is proposed by using motor state feedback for trajectory tracking of flexible joint robot with uncertain link dynamic model. Considering the link state information cannot be obtained, an observer is designed to estimate the link state information, and a dynamic surface controller is proposed based on link state observer. The controller based on the observer compared to backstepping controller avoids the repeated differentiation problem. At the same time, the dynamic surface method avoids the measurement of high order signal. The simulation results show that the designed controller has a good trajectory tracking effect, which effectively suppresses the residual vibration of the flexible joint robot. Moreover, the proposed controller and observer are robust to the uncertainty and external disturbance of the link dynamic model. The proposed controller can be directly applied in the flexible joint robot without installing additional sensors, which is very important for industrial applications.

\section{Introduction}

In recent years, not only are the requirements for robots in modern manufacturing limited to repetitive tasks, but also the robots can be quickly transformed in multiple tasks with the market demand changing from large batches, single mode to small batches and diversified directions[1]. Modern manufacturing thus calls for a flexible approach to manage production process, while the most flexible factor in a manufacturing process is human operators. In this instance, human and robot working collaboratively will be more efficient [2]. According to ISO 10218-2, collaborative robots are now allowed to work hand in hand with humans while being required to safely perform physical interactions in the dynamically changing and unstructured working environments [3]. Therefore, flexible joints are used extensively on collaborative robots, because when the flexible joint robot encounters obstacles during the operation, contact force between robot and obstacles may be relatively slight, and the robot can stop immediately. In [4], a robot with a variable stiffness joint is proposed, and the leaf spring is used to generate compliance. A servo-controller to asymptotically regulate the driving torque with unknown parameters of flexible robot is derived in [5].

Recently, many control methods of manipulator have been proposed such as adaptive neural network control [68], robust control [9], vibration control [10], and fuzzy control [11]. For the complex nonlinear systems whose model is uncertain, backstepping is a widely used method. But it suffers from the curse of dimension in the process of controller design. In order to avoid differentiating virtual control signals, dynamic surface control method is proposed in [12] by using first-order filters within the backstepping controller design. Currently, dynamic surface control method has been widely used in manipulators, marine vehicles, robots with flexible joints, and spacecrafts [13]. However, most studies on dynamic surface control focus on single-input singleoutput system [14]. Dynamic surface control is first applied to the controller design for nonlinear multi-input multioutput system like the flexible joint robot in [15]; however, the research is based on full-state feedback control, which needs to measure the link position, while most manipulators only 
have motor sensors installed, and the link state information cannot be acquired; therefore, this method cannot be used in industry directly. In practice, installing additional sensors can lead to a high cost design for the motion control system for industrial robots [16]. In order to obtain the link state information of the flexible joint manipulator, observer which can estimate the state variable unmeasurable is necessary. In [17], a neural network observer is designed to estimate the position and velocity of the link, but it is worth mentioning that this work is only used for single-input single-output system.

Generally, the dynamic model of a flexible joint manipulator can be divided into two parts: the link dynamic and the motor dynamic. Considering the parameters of motor are easy to obtain, it can be assumed that the motor dynamic model is precisely known. Since the parameters such as the quality of link are quite different from the actual values, and the quality of payload changes with the operation of manipulator, and the manipulator is sometimes subject to external disturbance, the observer, and the controller need to be robust. Therefore, in this paper, a controller based on dynamic surface control and observer is proposed by using motor state feedback for trajectory tracking of flexible joint robot with uncertain link dynamic model. Compared with existing results, the contribution of this paper is listed as follows. First, because the states of the link are nonmeasurable in industrial robot, a high-gain observer is proposed to estimate link positions and velocities. Since there is no need to install additional sensors, the proposed method will further facilitate its practical applications. Second, the trajectory tracking controller is designed for the multiple-input multiple-output system using the dynamic surface method considering the uncertain link dynamic model. It can be seen that the dynamic surface method ends the complexity arising due to the explosion of terms by introducing a first-order filter into the virtual control input at each backstepping design procedure, simplifies the controller design steps, and avoids the measurement of high-order signals. In addition, the designed controller can be adjusted by a limited number of parameters, which facilitates the debugging of the controller performance. Third, the system stability is analyzed by using Lyapunov stability analysis method, and simulation studies are performed to illustrate the theoretical results. The system stability analysis proves that the dynamic surface controller based on the state observer makes all signals in the closedloop system be UUB, and the first-order filter is introduced so that the tracking error no longer converges to zero, but can converge to small enough neighborhoods around the design parameters. It also proves that the designed controller based on observer is robust to the parameter uncertainty of the mass of the links and payload.

The paper is organized as follows: Section 2 states the problem formulation. A link observer is designed for the flexible joint robot considering the uncertainty and disturbance in Section 3. Section 4 presents the designed controller along with stability analysis. Section 5 provides simulation results to illustrate the theoretical results. Section 6 concludes this paper.

\section{Problem Formulation}

Consider a flexible joint robot, whose dynamics can be described as [18]

$$
\begin{array}{r}
D\left(q_{l}\right) \ddot{q}_{l}+C\left(q_{l}, \dot{q}_{l}\right) \dot{q}_{l}+G\left(q_{l}\right)+k\left(q_{l}-q_{r}\right)=0 \\
J \ddot{q}_{r}-k\left(q_{l}-q_{r}\right)=\tau
\end{array}
$$

where joints 1,2, and 3 are flexible joints, $q_{r} \in R^{3 \times 1}$ and $q_{l}$ $\in R^{3 \times 1}$ represent, respectively, the motor angles and link positions, $D\left(q_{l}\right) \in R^{3 \times 3}$ is the link inertia matrix, $C\left(q_{l}, \dot{q}_{l}\right) \dot{q}_{l} \in$ $R^{3 \times 1}$ represents the Coriolis and centrifugal forces, $G\left(q_{l}\right) \in$ $R^{3 \times 1}$ is the gravitational force vector, $k$ represents joint stiffness, $\tau \in R^{3 \times 1}$ represents torque, and $J \in R^{3 \times 3}$ is the matrix of the moments of the inertia of the motors.

Equations (1) and (2) have several fundamental properties which can be exploited to facilitate control system design. These properties are as follows.

Property 1. The link inertia matrix $D\left(q_{l}\right)$ is symmetric and positive definite, and both $D\left(q_{l}\right)$ and $D^{-1}\left(q_{l}\right)$ are uniformly bounded as follows: $\left\|D\left(q_{l}\right)\right\| \leq M_{D}$ and $\left\|D\left(q_{l}\right)^{-1}\right\| \leq M_{I D}$. Since $k$ is a constant matrix, $\left\|D\left(q_{l}\right)^{-1} k\right\| \leq M_{D k}$, where $M_{D}$, $M_{I D}$, and $M_{D k}$ are positive constants.

Property 2. $C\left(q_{l}, \dot{q}_{l}\right)$ is uniformly bounded as follows: $\left\|C\left(q_{l}, \dot{q}_{l}\right)\right\| \leq M_{C}$, where $M_{C}$ is a positive constant.

Property 3. The gravitational term $G\left(q_{l}\right)$ is uniformly bounded as follows: $\left\|G\left(q_{l}\right)\right\| \leq M_{G}$, where $M_{G}$ is a positive constant.

Property 4. The matrix $\dot{D}\left(q_{l}\right)-2 C\left(q_{l}, \dot{q}_{l}\right)$ is skew-symmetric.

Property 5. $J$ is symmetric, positive definite, $\|J\| \leq M_{J}$, where $M_{J}$ is a positive constant.

\section{Link State Observer}

For the flexible joint robot described in (1) and (2), a state observer for estimating the position and velocity of the link is designed by using the method in [19].

If we define $x_{1}=q_{l}, x_{2}=\dot{q}_{l}, x_{3}=q_{r}, x_{4}=\dot{q}_{r}$, the dynamic system (1) and (2) are described as

$$
\begin{aligned}
& \dot{x}_{1}=x_{2} \\
& \dot{x}_{2}=-D^{-1}\left(x_{1}\right)\left[C\left(x_{1}, x_{2}\right) x_{2}+G\left(x_{1}\right)+k\left(x_{1}-x_{3}\right)\right] \\
& \dot{x}_{3}=x_{4} \\
& J \dot{x}_{4}=\tau+k\left(x_{1}-x_{3}\right)
\end{aligned}
$$

It is assumed that the parameters in (3) are nominal parameters. When the parameters of the link dynamic model are uncertain and there is external disturbance, the real state equation can be expressed as follows:

$$
\begin{aligned}
& \dot{x}_{1}=x_{2} \\
& \dot{x}_{2}
\end{aligned}
$$




$$
\begin{aligned}
= & -D^{-1}\left(x_{1}\right)\left[C\left(x_{1}, x_{2}\right) x_{2}+G\left(x_{1}\right)+k\left(x_{1}-x_{3}\right)\right] \\
& +E_{u d} \\
\dot{x}_{3}= & x_{4} \\
J \dot{x}_{4}= & \tau+k\left(x_{1}-x_{3}\right)
\end{aligned}
$$

where $E_{u d}$ is the uncertainty and external disturbance, the specific form of which is as follows:

$$
\begin{aligned}
E_{u d} & \left(x_{1}, x_{2}, x_{3}\right)=-\bar{D}^{-1}\left(x_{1}\right) \\
\cdot & {\left[\bar{C}\left(x_{1}, x_{2}\right) x_{2}+\bar{G}\left(x_{1}\right)+k\left(x_{1}-x_{3}\right)+\tau_{d l}\right] } \\
& +D^{-1}\left(x_{1}\right)\left[C\left(x_{1}, x_{2}\right) x_{2}+G\left(x_{1}\right)+k\left(x_{1}-x_{3}\right)\right]
\end{aligned}
$$

where $\bar{D}\left(x_{1}\right), \bar{C}\left(x_{1}, x_{2}\right) x_{2}, \bar{G}\left(x_{1}\right)$ are real parameters, $\tau_{d l}$ is the external disturbance, and $E_{u d}$ is bounded as follows: $E_{u d} \leq E_{u d}^{*}, E_{u d}^{*}$ is a constant.

It is assumed that $q_{r}$ and $\dot{q}_{r}$ are measurable. The two outputs are defined as follows:

$$
\begin{aligned}
& y_{1}=x_{3} \\
& y_{2}=x_{4}
\end{aligned}
$$

Now consider the following change of coordinates:

$$
\begin{aligned}
& z_{1}=k^{-1} J x_{4} \\
& z_{2}=x_{1} \\
& z_{3}=x_{2}
\end{aligned}
$$

The reduced order system obtained via the above change of coordinates satisfies

$$
\begin{aligned}
\dot{z}_{1} & =k^{-1} J x_{4}=z_{2}+k^{-1} \tau-y_{1} \\
\dot{z}_{2} & =\dot{x}_{1}=x_{2}=z_{3} \\
\dot{z}_{3} & =\dot{x}_{2} \\
& =-D^{-1}\left(z_{2}\right)\left[C\left(z_{2}, z_{3}\right) z_{3}+G\left(z_{2}\right)+k\left(z_{2}-y_{1}\right)\right]
\end{aligned}
$$

Define $\phi\left(z_{2}, z_{3}, y_{1}\right)=-D^{-1}\left(z_{2}\right)\left[C\left(z_{2}, z_{3}\right) z_{3}+G\left(z_{2}\right)+k\left(z_{2}-y_{1}\right)\right]$. Equations (8), (9), and (10) can be expressed as follows:

$$
\begin{aligned}
\dot{z} & =A z+\Phi\left(z, y_{1}, \tau\right) \\
y_{2} & =J^{-1} k C z
\end{aligned}
$$

where

$$
\begin{array}{r}
A \in R^{9 \times 9}=\left[\begin{array}{lll}
0 & I & 0 \\
0 & 0 & I \\
0 & 0 & 0
\end{array}\right] \\
C \in R^{3 \times 9}=\left[\begin{array}{lll}
I & 0 & 0
\end{array}\right] \\
\Phi\left(z, y_{1}, \tau\right)=\left[\begin{array}{c}
k^{-1} \tau-y_{1} \\
0 \\
\phi\left(z_{2}, z_{3}, y_{1}\right)
\end{array}\right]
\end{array}
$$

Define $\bar{H}=H k^{-1} J$; (11) can be expressed as follows:

$$
\begin{aligned}
\dot{\bar{z}} & =A \widehat{z}+\Phi\left(\widehat{z}, y_{1}, \tau\right)+\Delta \bar{H}\left(y_{2}-C \widehat{z}\right) \\
& =A \widehat{z}+\Phi\left(\widehat{z}, y_{1}, \tau\right)+\Delta H C(z-\widehat{z})
\end{aligned}
$$

Choose a matrix $H$ that places all the eigenvalues of $(A-H C)$ in the left half of the complex plane. $\mathrm{H} \in R^{9 \times 3}=\left[H_{1}^{T}\right.$ । $\left.H_{2}^{T} H_{3}^{T}\right]^{T}$ and $H_{i} \in R^{3 \times 3}, i=1,2,3$

The gain matrix can be expressed as $\Delta \in R^{9 \times 9}=$ $\left[\begin{array}{ccc}\Gamma I & 0 & 0 \\ 0 & \Gamma^{2} I & 0 \\ 0 & 0 & \Gamma^{3} I\end{array}\right], \Gamma \in R^{3 \times 3}=\operatorname{diag}\left\{\Gamma_{1}, \Gamma_{2}, \Gamma_{3}\right\}$, and $\Gamma_{1} \geq 1, \Gamma_{2} \geq 1$, $\Gamma_{3} \geq 1$.

Consider the real dynamic system (4), $\bar{\phi}\left(\widehat{z}_{2}, \widehat{z}_{3}, y_{1}\right)=$ $\phi\left(\widehat{z}_{2}, \widehat{z}_{3}, y_{1}\right)+E_{u d} ; \Phi$ can be expressed as follows:

$$
\begin{aligned}
\Phi\left(\widehat{z}, y_{1}, \tau\right) & =\left[\begin{array}{c}
k^{-1} \tau-y_{1} \\
0 \\
\phi\left(\widehat{z}_{2}, \widehat{z}_{3}, y_{1}\right)
\end{array}\right] \\
& =\left[\begin{array}{c}
k^{-1} \tau-y_{1} \\
0 \\
\bar{\phi}\left(\widehat{z}_{2}, \widehat{z}_{3}, y_{1}\right)-E_{u d}
\end{array}\right]
\end{aligned}
$$

Substituting (7) into (13) yields the equations of observer.

$$
\begin{aligned}
\dot{\hat{x}}_{1}= & \widehat{x}_{2}+\Gamma^{2} H_{2} k^{-1} J\left(x_{4}-\widehat{x}_{4}\right) \\
\dot{\hat{x}}_{2}= & -D^{-1}\left(\widehat{x}_{1}\right)\left[C\left(\widehat{x}_{1}, \widehat{x}_{2}\right) x_{2}+G\left(\widehat{x}_{1}\right)\right. \\
& +k\left(x_{1}-x_{3}\right)-\Gamma^{3} H_{3} k^{-1} J\left(x_{4}-\widehat{x}_{4}\right) \\
k^{-1} J \dot{\hat{x}}_{4}= & \widehat{x}_{1}+k^{-1} \tau-x_{3}+\Gamma H_{1} k^{-1} J\left(x_{4}-\widehat{x}_{4}\right)
\end{aligned}
$$

Define $\widetilde{x}_{i}=x_{i}-\widehat{x}_{i}$; the observer state error can be expressed as follows:

$$
\begin{aligned}
& \dot{\tilde{x}}_{1}= \widetilde{x}_{2}-\Gamma^{2} H_{2} k^{-1} J \tilde{x}_{4} \\
& \dot{\tilde{x}}_{2} \\
&=-D^{-1}\left(x_{1}\right)\left[C\left(x_{1}, x_{2}\right) x_{2}+G\left(x_{1}\right)+k\left(x_{1}-x_{3}\right)\right] \\
&+E_{u d} \\
&+D^{-1}\left(\widehat{x}_{1}\right)\left[C\left(\widehat{x}_{1}, \widehat{x}_{2}\right) x_{2}+G\left(\widehat{x}_{1}\right)+k\left(x_{1}-x_{3}\right)\right] \\
& \quad \Gamma^{3} H_{3} k^{-1} J \widetilde{x}_{4} \\
&= \bar{\phi}\left(x_{1}, x_{2}, x_{3}\right)-\phi\left(\widehat{x}_{1}, \widehat{x}_{2}, x_{3}\right)-\Gamma^{3} H_{3} k^{-1} J \tilde{x}_{4} \\
& k^{-1} J \dot{\tilde{x}}_{4}=\widetilde{x}_{1}-\Gamma H_{1} k^{-1} J \widetilde{x}_{4}
\end{aligned}
$$

\section{Dynamic Surface Controller Based on Observer}

A visualization of the proposed controller structure is shown in Figure 1. In this section, we assume we cannot measure the position $x_{1}$ and the velocity $x_{2}$ of the link. We use the observer proposed in the previous section to estimate the position $x_{1}$ and the velocity $x_{2}$. 


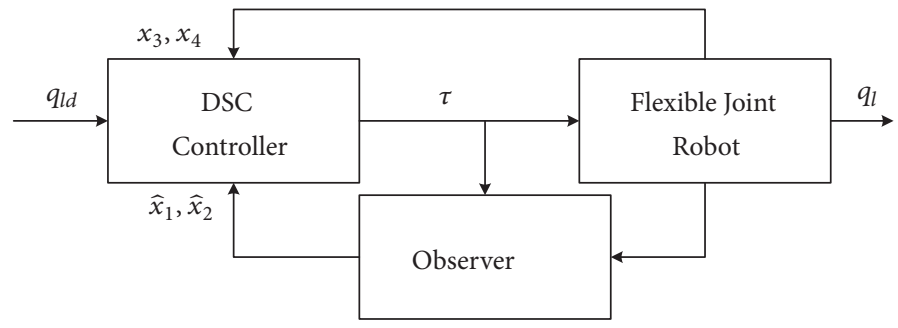

FIGURE 1: Controller structure.

4.1. Controller Design. First, we let $S_{1}=\widehat{x}_{1}-q_{l d}$ and compute its derivative; we have

$$
\dot{S}_{1}=\dot{\hat{x}}_{1}-\dot{q}_{l d}=\widehat{x}_{2}-\dot{q}_{l d}+\Gamma^{2} H_{2} k^{-1} J \tilde{x}_{4}
$$

Define the virtual control variable as follows:

$$
\bar{x}_{2}=-\eta_{1} S_{1}+\dot{q}_{l d}-\Gamma^{2} H_{2} k^{-1} J \tilde{x}_{4}
$$

where $\eta_{1}>0$ is the parameter of controller.

Then we let $\bar{x}_{2}$ pass through a first-order filter, we obtain a new variable $x_{2 f}$, and the filter is expressed as follows:

$$
\begin{aligned}
\alpha_{2} \dot{x}_{2 f}+x_{2 f} & =\bar{x}_{2} \\
x_{2 f}(0) & =\bar{x}_{2}(0)
\end{aligned}
$$

where $\alpha_{2}$ is the time constant.

Second, we let $S_{2}=\widehat{x}_{2}-x_{2 f}$ and compute its derivative; we have

$$
\begin{aligned}
\dot{S}_{2}= & \dot{\hat{x}}_{2}-\dot{x}_{2 f} \\
= & -D^{-1}\left(\hat{x}_{1}\right)\left[C\left(\widehat{x}_{1}, \widehat{x}_{2}\right) x_{2}+G\left(\hat{x}_{1}\right)+k\left(\widehat{x}_{1}-x_{3}\right)\right] \\
& +\Gamma^{3} H_{3} k^{-1} J \tilde{x}_{4}-\frac{\left(\bar{x}_{2}-x_{2 f}\right)}{\alpha_{2}}
\end{aligned}
$$

Define the virtual control variable as follows:

$$
\begin{aligned}
\bar{x}_{3} & =k^{-1}\left[k \hat{x}_{1}+C\left(\hat{x}_{1}, \widehat{x}_{2}\right) \hat{x}_{2}+G\left(\hat{x}_{1}\right)\right. \\
& \left.+D\left(\hat{x}_{1}\right)\left(-\eta_{2} S_{2}-\Gamma^{3} H_{3} k^{-1} J \tilde{x}_{4}+\frac{\left(\bar{x}_{2}-x_{2 f}\right)}{\alpha_{2}}\right)\right]
\end{aligned}
$$

where $\eta_{2}>0$ is the parameter of controller.

Then we let $\bar{x}_{3}$ pass through a first-order filter, we obtain a new variable $x_{3 f}$, and we have

$$
\begin{aligned}
\alpha_{3} \dot{x}_{3 f}+x_{3 f} & =\bar{x}_{3}, \\
x_{3 f}(0) & =\bar{x}_{3}(0)
\end{aligned}
$$

where $\alpha_{3}$ is the time constant. have

Third, we let $S_{3}=x_{3}-x_{3 f}$ and compute its derivative; we

$$
\dot{S}_{3}=\dot{x}_{3}-\dot{x}_{3 f}=x_{4}-\frac{\left(\bar{x}_{3}-x_{3 f}\right)}{\alpha_{3}}
$$

Define the virtual control variable as follows:

$$
\bar{x}_{4}=-\eta_{3} S_{3}+\frac{\left(\bar{x}_{3}-x_{3 f}\right)}{\alpha_{3}}
$$

where $\eta_{3}>0$ is the parameter of controller.

Then we let $\bar{x}_{4}$ pass through a first-order filter, we obtain a new varialbe $x_{4 f}$, and we have

$$
\begin{aligned}
\alpha_{4} \dot{x}_{4 f}+x_{4 f} & =\bar{x}_{4}, \\
x_{4 f}(0) & =\bar{x}_{4}(0)
\end{aligned}
$$

where $\alpha_{4}$ is the time constant.

Fourth, we let $S_{4}=x_{4}-x_{4 f}$ and compute its derivative; we have

$$
\begin{aligned}
\dot{S}_{4} & =\dot{x}_{4}-\dot{x}_{4 f}=J^{-1}\left[\tau+k\left(x_{1}-x_{3}\right)\right]-\frac{\left(\bar{x}_{4}-x_{4 f}\right)}{\alpha_{4}} \\
& =J^{-1}\left[\tau+k \widehat{x}_{1}-k x_{3}+k \tilde{x}_{1}\right]-\frac{\left(\bar{x}_{4}-x_{4 f}\right)}{\alpha_{4}}
\end{aligned}
$$

Choose the control input as follows:

$$
\tau=k x_{3}-k \widehat{x}_{1}+J\left[\frac{\left(\bar{x}_{4}-x_{4 f}\right)}{\alpha_{4}}-\eta_{4} S_{4}\right]
$$

where $\eta_{4}>0$ is the parameter of controller.

\subsection{Stability Analysis}

Assumption 6. The position and velocity of the link are bounded.

Assumption 7. The uncertainty and external disturbance of the model are bounded.

Assumption 8. $\hat{x}_{1}, \widehat{x}_{2}$ are bounded.

Property 9. $\Delta, \Gamma$ are positive definite diagonal matrix and $\|\Gamma\|_{2} \leq \lambda_{\max }(\Gamma),-\|\Gamma\|_{2} \leq-\lambda_{\min }(\Gamma)$.

Property 10. The eigenvalues of $\Gamma$ satisfy these conditions: $\lambda_{\text {max }}\left(\Gamma^{m}\right)=\lambda_{\max }^{m}(\Gamma), \lambda_{\min }\left(\Gamma^{m}\right)=\lambda_{\min }^{m}(\Gamma)$ where $m$ is an integer, $\lambda_{\min }(\cdot)$ denotes the minimum eigenvalue, and $\lambda_{\max }(\cdot)$ denotes the maximum eigenvalue.

Define the boundary layer error $r_{i}=x_{i f}-\bar{x}_{i}$ and we have

$$
r_{2}=x_{2 f}-\bar{x}_{2}=x_{2 f}+\eta_{1} S_{1}-\dot{q}_{l d}+\Gamma^{2} H_{2} k^{-1} J \tilde{x}_{4}
$$




$$
\begin{aligned}
r_{3} & =x_{3 f}-\bar{x}_{3}=x_{3 f}-k^{-1}\left[\mathrm{k} \widehat{\mathrm{x}}_{1}+\mathrm{C}\left(\widehat{\mathrm{x}}_{1}, \widehat{\mathrm{x}}_{2}\right) \widehat{\mathrm{x}}_{2}\right. \\
& +\mathrm{G}\left(\widehat{\mathrm{x}}_{1}\right) \\
& \left.+\mathrm{D}\left(\widehat{\mathrm{x}}_{1}\right)\left(-\eta_{2} \mathrm{~S}_{2}+\Gamma^{3} H_{3} k^{-1} J \tilde{x}_{4}+\frac{\left(\bar{x}_{2}-x_{2 f}\right)}{\alpha_{2}}\right)\right] \\
& =x_{3 f}-k^{-1}\left[\mathrm{k} \widehat{\mathrm{x}}_{1}+\mathrm{C}\left(\widehat{\mathrm{x}}_{1}, \widehat{\mathrm{x}}_{2}\right) \widehat{\mathrm{x}}_{2}+\mathrm{G}\left(\widehat{\mathrm{x}}_{1}\right)\right. \\
& \left.+\mathrm{D}\left(\widehat{\mathrm{x}}_{1}\right)\left(-\eta_{2} \mathrm{~S}_{2}-\frac{r_{2}}{\alpha_{2}}\right)\right] \\
r_{4} & =x_{4 f}-\bar{x}_{4}=x_{4 f}+\eta_{3} S_{3}-\frac{\left(\bar{x}_{3}-x_{3 f}\right)}{\alpha_{3}}=x_{4 f} \\
& +\eta_{3} S_{3}+\frac{r_{3}}{\alpha_{3}}
\end{aligned}
$$

Now, $\dot{S}_{1}$ can be expressed as

$$
\begin{aligned}
\dot{S}_{1} & =S_{2}+x_{2 f}-\dot{q}_{l d}+\Gamma^{2} H_{2} k^{-1} J \tilde{x}_{4} \\
& =S_{2}+r_{2}-\eta_{1} S_{1}+\Gamma^{2} H_{2} k^{-1} J \tilde{x}_{4}
\end{aligned}
$$

$\dot{S}_{2}$ can be expressed as

$\dot{S}_{2}$

$$
\begin{aligned}
= & -D^{-1}\left(\widehat{x}_{1}\right)\left[C\left(\widehat{x}_{1}, \widehat{x}_{2}\right) \widehat{x}_{2}+G\left(\widehat{x}_{1}\right)+k\left(\widehat{x}_{1}-x_{3}\right)\right] \\
& +\Gamma^{3} H_{3} k^{-1} J \tilde{x}_{4}-\frac{\left(\bar{x}_{2}-x_{2 f}\right)}{\alpha_{2}} \\
= & D^{-1}\left(\hat{x}_{1}\right)\left[k\left(r_{3}+S_{3}\right)\right]-\eta_{2} S_{2}+\Gamma^{3} H_{3} k^{-1} J \tilde{x}_{4}
\end{aligned}
$$

$\dot{S}_{3}$ can be expressed as

$$
\begin{aligned}
\dot{S}_{3} & =x_{4}-\frac{\left(\bar{x}_{3}-x_{3 f}\right)}{\alpha_{3}}=S_{4}+x_{4 f}-\frac{\left(\bar{x}_{3}-x_{3 f}\right)}{\alpha_{3}} \\
& =S_{4}+r_{4}-\eta_{3} S_{3}
\end{aligned}
$$

$\dot{S}_{4}$ can be expressed as

$$
\dot{S}_{4}=-\eta_{4} S_{4}+J^{-1} k \tilde{x}_{1}
$$

The derivatives of $r_{i}$ are

$$
\begin{aligned}
& \dot{r}_{2}=-\frac{r_{2}}{\alpha_{2}}-\Xi_{2}\left(S_{1}, S_{2}, r_{2}, Q_{l d}\right) \\
& \dot{r}_{3}=-\frac{r_{3}}{\alpha_{3}}-\Xi_{3}\left(S_{1}, S_{2}, S_{3}, r_{2}, r_{3}, Q_{l d}\right) \\
& \dot{r}_{4}=-\frac{r_{4}}{\alpha_{4}}-\Xi_{4}\left(S_{1}, S_{2}, S_{3}, S_{4}, r_{2}, r_{3}, r_{4}, Q_{l d}\right)
\end{aligned}
$$

where

$$
\begin{aligned}
& Q_{l d}=\left[\begin{array}{lll}
q_{l d}^{T} & \dot{q}_{l d}^{T} & \ddot{q}_{l d}^{T}
\end{array}\right]^{T} \\
& \Xi_{2}\left(S_{1}, S_{2}, r_{2}, \dot{\tilde{x}}_{4}, Q_{l d}\right)=-\eta_{1} \dot{S}_{1}+\ddot{q}_{l d}+\Gamma^{2} H_{2} k^{-1} J \dot{\tilde{x}}_{4} \\
& \Xi_{3}\left(S_{1}, S_{2}, S_{3}, r_{2}, r_{3}, \tilde{x}_{1}, \tilde{x}_{2}, \tilde{x}_{4}, Q_{l d}\right)=k^{-1}\left[k \dot{\bar{x}}_{1}\right.
\end{aligned}
$$

$$
\begin{aligned}
& +\left(\dot{\hat{x}}_{1}^{T} \frac{\partial C}{\hat{x}_{1}}+\dot{\hat{x}}_{2}^{T} \frac{\partial C}{\hat{x}_{2}}\right) \widehat{x}_{2}+C\left(\widehat{x}_{1}, \widehat{x}_{2}\right) \dot{\hat{x}}_{2}+\frac{\partial C}{\hat{x}_{1}} \dot{\hat{x}}_{1} \\
& \left.+\dot{\hat{x}}_{1}^{T} \frac{\partial C}{\hat{x}_{1}}-\eta_{2} S_{2}-\frac{r_{2}}{\alpha_{2}}+E_{u d}\right]+D\left(\widehat{x}_{1}\right)\left(-\eta_{2} \dot{S}_{2}\right. \\
& \left.-\frac{\dot{r}_{2}}{\alpha_{2}}+E_{u d}\right) \\
& \Xi_{4}\left(S_{1}, S_{2}, S_{3}, S_{4}, r_{2}, r_{3}, r_{4}, \widetilde{x}_{1}, \widetilde{x}_{2}, \tilde{x}_{4}, Q_{l d}\right)=-\eta_{3} \dot{S}_{3} \\
& -\frac{\dot{r}_{3}}{\alpha_{3}} .
\end{aligned}
$$

Now, we choose a Lyapunov function:

$$
V=V_{c}+V_{o}
$$

where $V_{c}$ is the Lyapunov function of controller and $V_{o}$ is the Lyapunov function of observer.

$$
V_{c}=\frac{1}{2}\left[\sum_{i=1}^{4} S_{i}^{T} S_{i}+\sum_{i=1}^{3} r_{i+1}^{T} r_{i+1}\right]
$$

Define $\tilde{x}=\Delta^{-1}\left[\begin{array}{lll}\mathrm{k}^{-1} \mathrm{~J} \tilde{x}_{4} & \tilde{x}_{1} & \tilde{x}_{2}\end{array}\right]^{T}$. Given a positive definite diagonal matrix $\Lambda=\operatorname{diag}\{\Gamma, \Gamma, \Gamma\}$ the derivative of $\tilde{x}$ can be expressed as

$$
\begin{aligned}
& \dot{\tilde{x}}=\Lambda(A-H C) \tilde{x}
\end{aligned}
$$

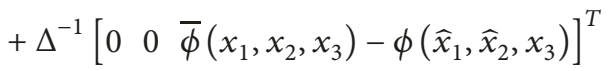

Let $\Omega$ be the solution to the equation $(A-H C)^{T} \Omega+\Omega(A-$ $H C)=-I$.

The Lyapunov function of observer can be expressed as

$$
V_{o}=\frac{1}{2} \tilde{x}^{T} \Lambda^{3} \Omega \tilde{x}
$$

Using (35) to (41), the time derivative of $V_{c}$ is

$$
\begin{aligned}
\dot{V}_{c}= & S_{1}^{T}\left(\mathrm{~S}_{2}+r_{2}-\eta_{1} \mathrm{~S}_{1}\right) \\
& +S_{2}^{T}\left[\mathrm{D}^{-1}\left(\widehat{\mathrm{x}}_{1}\right)\left(\mathrm{k}\left(\mathrm{r}_{3}+\mathrm{S}_{3}\right)\right)-\eta_{2} \mathrm{~S}_{2}\right] \\
& +S_{3}^{T}\left(\mathrm{~S}_{4}+r_{4}-\eta_{3} \mathrm{~S}_{3}\right)+\mathrm{S}_{4}^{T}\left(-\eta_{4} \mathrm{~S}_{4}+J^{-1} \mathrm{k} \widetilde{\mathrm{x}}_{1}\right) \\
& +\sum_{i=1}^{3} r_{i+1}^{T}\left(-\frac{1}{\alpha_{i+1}} \mathrm{r}_{i+1}+\Xi_{i+1}\right) \\
\leq & \left\|S_{1}\right\|\left\|S_{2}\right\|+\left\|S_{1}\right\|\left\|r_{2}\right\|-\eta_{1}\left\|S_{1}\right\|^{2} \\
& +\left\|D^{-1}\left(\widehat{\mathrm{x}}_{1}\right) \mathrm{k}\right\| S_{2}\left\|S_{2}\right\|\left(\left\|r_{3}\right\|+\left\|S_{3}\right\|\right)-\eta_{2}\left\|S_{2}\right\|^{2} \\
& +\left\|S_{3}\right\|\left\|S_{4}\right\|+\left\|S_{3}\right\|\left\|r_{4}\right\|-\eta_{3}\left\|S_{3}\right\|^{2}-\eta_{4}\left\|S_{4}\right\|^{2} \\
& +\left\|J^{-1} k\right\|_{2}\left\|S_{4}\right\|\left\|\widetilde{x}_{1}\right\| \\
& +\sum_{i=1}^{3}\left(-\frac{1}{\alpha_{i+1}}\left\|r_{i+1}\right\|+\left\|r_{i+1}\right\|\left\|\Xi_{i+1}\right\|\right)
\end{aligned}
$$


According to Property 1, applying Young's inequality to (47), we obtain

$$
\begin{aligned}
\dot{V}_{c} \leq & \left\|S_{1}\right\|^{2}+\frac{\left\|S_{2}\right\|^{2}}{4}+\left\|S_{1}\right\|^{2}+\frac{\left\|r_{2}\right\|^{2}}{4} \\
& +M_{D k}\left(\left\|S_{2}\right\|^{2}+\frac{\left\|r_{3}\right\|^{2}}{4}\right) \\
& +M_{D k}\left(\left\|S_{2}\right\|^{2}+\frac{\left\|S_{3}\right\|^{2}}{4}\right)+\left\|S_{3}\right\|^{2}+\frac{\left\|S_{4}\right\|^{2}}{4} \\
& +\left\|S_{3}\right\|^{2}+\frac{\left\|r_{4}\right\|^{2}}{4} \\
& +\lambda_{\max }\left(J^{-1} \mathrm{k}\right)\left(\left\|S_{4}\right\|^{2}+\frac{\left\|\tilde{x}_{1}\right\|^{2}}{4}\right)-\sum_{i=1}^{4} \eta_{i}\left\|S_{i}\right\|^{2} \\
& +\sum_{i=1}^{3}\left(-\frac{1}{\alpha_{i+1}}\left\|r_{i+1}\right\|^{2}+\left\|r_{i+1}\right\|^{2}+\frac{\left\|\Xi_{i+1}\right\|^{2}}{4}\right) \\
= & 2\left\|S_{1}\right\|^{2}+\left(\frac{1}{4}+2 M_{D k}\right)\left\|S_{2}\right\|^{2} \\
& +\left(2+\frac{M_{D k}}{4}\right)\left\|S_{3}\right\|^{2}+\left(\frac{1}{4}+\lambda_{\max }\left(J^{-1} \mathrm{k}\right)\right)\left\|S_{4}\right\|^{2} \\
& -\sum_{i=1}^{4} \eta_{1}\left\|S_{4}\right\|^{2}+\frac{5\left\|r_{2}\right\|^{2}}{4}+\frac{4+M_{D k}}{4}\left\|r_{3}\right\|^{2} \\
& +\frac{5\left\|r_{4}\right\|^{2}}{4}+\sum_{i=1}^{3}\left(-\frac{1}{\alpha_{i+1}}\left\|r_{i+1}\right\|^{2}+\frac{\left\|\Xi_{i+1}\right\|^{2}}{4}\right) \\
& +\frac{\lambda_{\max }\left(J^{-1} \mathrm{k}\right)}{4}\left\|\tilde{x}_{1}\right\|^{2} \\
&
\end{aligned}
$$

where $\lambda_{\max }(\cdot)$ denotes the maximum eigenvalue. Let

$$
\begin{aligned}
\eta_{1}^{*} & =\eta_{1}-2, \\
\eta_{2}^{*} & =\eta_{2}-\frac{1}{4}-2 M_{D k}, \\
\eta_{3}^{*} & =\eta_{3}-2-\frac{M_{D k}}{4}, \\
\eta_{4}^{*} & =\eta_{4}-\frac{1}{4}-\lambda_{\max }\left(J^{-1} k\right), \\
\frac{1}{\alpha_{2}^{*}} & =\frac{1}{\alpha_{2}}-\frac{5}{4}, \\
\frac{1}{\alpha_{3}^{*}} & =\frac{1}{\alpha_{3}}-\frac{4+M_{D k}}{4}, \\
\frac{1}{\alpha_{4}^{*}} & =\frac{1}{\alpha_{4}}-\frac{5}{4},
\end{aligned}
$$

and we have

$$
\begin{aligned}
\dot{V}_{c} \leq & -\sum_{i=1}^{4} \eta_{i}^{*}\left\|S_{i}\right\|^{2}-\sum_{i=2}^{3} \frac{1}{\alpha_{i+1}^{*}}\left\|r_{i+1}\right\|^{2}+\sum_{i=2}^{3} \frac{\left\|\Xi_{i+1}\right\|^{2}}{4} \\
& +\frac{\lambda_{\max }\left(J^{-1} k\right)}{4}\left\|\tilde{x}_{1}\right\|^{2}
\end{aligned}
$$

According to Property 9, it follows that

$$
\begin{aligned}
\dot{V}_{c} \leq & -\sum_{i=1}^{4} \eta_{i}^{*}\left\|S_{i}\right\|^{2}-\sum_{i=2}^{3} \frac{1}{\alpha_{i+1}^{*}}\left\|r_{i+1}\right\|^{2}+\sum_{i=2}^{3} \frac{\left\|\Xi_{i+1}\right\|^{2}}{4} \\
& +\frac{\lambda_{\max }\left(J^{-1} k\right)}{4}\left\|\Gamma^{2}\right\|\left\|_{2}\right\| \Gamma^{-2} \tilde{x}_{1} \|^{2} \\
\leq & -\sum_{i=1}^{4} \eta_{i}^{*}\left\|S_{i}\right\|^{2}-\sum_{i=2}^{3} \frac{1}{\alpha_{i+1}^{*}}\left\|r_{i+1}\right\|^{2}+\sum_{i=2}^{3} \frac{\left\|\Xi_{i+1}\right\|^{2}}{4} \\
& +\frac{\lambda_{\max }\left(J^{-1} k\right)}{4} \lambda_{\max }\left(\Gamma^{2}\right)\|\tilde{x}\|^{2}
\end{aligned}
$$

Using (14) to (20), the time derivative of $V_{o}$ is

$$
\begin{aligned}
& \dot{V}_{o}=\frac{1}{2}\left(\dot{\tilde{\mathrm{x}}}^{T} \Lambda^{3} \Omega \widetilde{\mathrm{x}}+\widetilde{\mathrm{x}}^{T} \Lambda^{3} \Omega \dot{\tilde{\mathrm{x}}}\right) \\
& =\frac{1}{2}\{\Lambda(\mathrm{A} \\
& \text { - HC) } \left.\tilde{\mathrm{x}}+\Delta^{-1}\left[\begin{array}{lll}
0 & 0 & \bar{\phi}\left(x_{1}, x_{2}, x_{3}\right)-\phi\left(\widehat{x}_{1}, \widehat{x}_{2}, x_{3}\right)
\end{array}\right]^{T}\right\}^{T} \Lambda^{3} \Omega \tilde{x} \\
& +\frac{1}{2} \Lambda^{3} \tilde{x}^{T} \Omega\{\Lambda(\mathrm{A} \\
& \text { - HC) } \left.\tilde{\mathrm{x}}+\Delta^{-1}\left[\begin{array}{lll}
0 & 0 & \bar{\phi}\left(x_{1}, x_{2}, x_{3}\right)-\phi\left(\widehat{x}_{1}, \widehat{x}_{2}, x_{3}\right)
\end{array}\right]^{T}\right\}^{T} \tilde{x}=\frac{1}{2} \Lambda^{4} \tilde{x}^{T} \widetilde{x} \\
& +\frac{1}{2}
\end{aligned}
$$

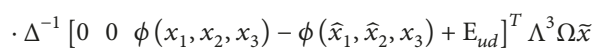

$$
\begin{aligned}
& +\frac{1}{2}
\end{aligned}
$$

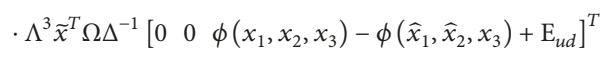

where $\phi\left(x_{1}, x_{2}, x_{3}\right)-\phi\left(\widehat{x}_{1}, \hat{x}_{2}, x_{3}\right)$ can be expressed as

$$
\begin{aligned}
\| \phi & \left(x_{1}, x_{2}, x_{3}\right)-\phi\left(\hat{x}_{1}, \hat{x}_{2}, x_{3}\right) \| \\
\leq & \left\|D^{-1}\left(x_{1}\right) k\left(x_{3}-x_{1}\right)-D^{-1}\left(\widehat{x}_{1}\right) k\left(x_{3}-\widehat{x}_{1}\right)\right\| \\
& +\left\|D^{-1}\left(\widehat{x}_{1}\right) C\left(\widehat{x}_{1}, \hat{x}_{2}\right)-D^{-1}\left(x_{1}\right) C\left(x_{1}, x_{2}\right) x_{2}\right\| \\
& +\left\|D^{-1}\left(\widehat{x}_{1}\right) G\left(\widehat{x}_{1}\right)-D^{-1}\left(x_{1}\right) G\left(x_{1}\right)\right\|
\end{aligned}
$$

According to Property 1 and Assumptions 6, 7, and 8, we have

$$
\begin{aligned}
& \left\|D^{-1}\left(x_{1}\right) k\left(x_{3}-x_{1}\right)-D^{-1}\left(\widehat{x}_{1}\right) k\left(x_{3}-\widehat{x}_{1}\right)\right\| \\
& \quad \leq \| D^{-1}\left(x_{1}\right) k\left(x_{3}-x_{1}\right)+D^{-1}\left(x_{1}\right) k x_{1} \\
& -D^{-1}\left(x_{1}\right) k \widehat{x}_{1}-D^{-1}\left(\widehat{\mathrm{r}}_{2}\right) k\left(\mathrm{x}_{3}-\widehat{\mathrm{x}}_{1}\right) \| \\
& \quad \leq\left\|D^{-1}\left(x_{1}\right) k\left(x_{1}-\widehat{x}_{1}\right)\right\|+\| D^{-1}\left(x_{1}\right) \\
& -D^{-1}\left(\widehat{x}_{1}\right)\|\|_{2}\left\|k\left(\mathrm{x}_{3}-\widehat{x}_{1}\right)\right\| \leq\left\|D^{-1}\left(x_{1}\right) k\right\|_{2} \| x_{1} \\
& -\widehat{x}_{1}\left\|+\varsigma_{1}\right\| k\left\|_{2}\right\| D^{-1}\left(x_{1}\right)-D^{-1}\left(\widehat{x}_{1}\right)\left\|\leq M_{D K}\right\| \mathrm{x}_{1} \\
& -\widehat{x}_{1}\left\|+\varsigma_{1} \lambda_{\max }(\mathrm{k})\right\| D^{-1}\left(x_{1}\right)-D^{-1}\left(\widehat{x}_{1}\right)\left\|\leq \varsigma_{2}\right\| x_{1} \\
& -\widehat{x}_{1}\left\|=\varsigma_{2}\right\| \widetilde{x}_{1} \|
\end{aligned}
$$


where $\lambda_{\max }(\mathrm{k})$ denotes the maximum eigenvalues of $k, \varsigma_{1}$, and $\varsigma_{2}$ are all positive constant, and $\left\|x_{3}-\widehat{x}_{1}\right\| \leq \varsigma_{1}$.

In the same way, we can get

$$
\begin{aligned}
& \left\|D^{-1}\left(\widehat{x}_{1}\right) C\left(\widehat{x}_{1}, \widehat{x}_{2}\right) \dot{\hat{x}}_{2}-D^{-1}\left(x_{1}\right) C\left(x_{1}, x_{2}\right) x_{2}\right\| \\
& \quad \leq \varsigma_{3}\left\|\widetilde{x}_{2}\right\|+\varsigma_{4}\left\|\tilde{x}_{1}\right\| \\
& \left\|D^{-1}\left(\widehat{x}_{1}\right) G\left(\widehat{x}_{1}\right)-D^{-1}\left(x_{1}\right) G\left(x_{1}\right)\right\| \leq \varsigma_{5}\left\|\tilde{x}_{1}\right\|
\end{aligned}
$$

where $\varsigma_{3}, \varsigma_{4}, \varsigma_{5}$ are all positive constants.

And then, we have

$$
\left\|\phi\left(x_{1}, x_{2}, x_{3}\right)-\phi\left(\hat{x}_{1}, \hat{x}_{2}, x_{3}\right)\right\| \leq \varsigma_{6}\left\|\tilde{x}_{1}\right\|+\varsigma_{7}\left\|\tilde{x}_{2}\right\|
$$

where $\varsigma_{6}, \varsigma_{7}$ are positive constants.

Applying (57) to (52), we can get

$$
\begin{aligned}
\dot{V}_{o} \leq & -\frac{1}{2} \lambda_{\min }\left(\Gamma^{4}\right)\|\tilde{x}\|^{2}+\lambda_{\max }\left(\Gamma^{3}\right)\|\Omega\|_{2}\left\|\Gamma^{-3}\right\|_{2} \\
\cdot & {\left[\|\tilde{x}\|\left(\varsigma_{6}\left\|\tilde{x}_{1}\right\|+\varsigma_{7}\left\|\tilde{x}_{2}\right\|\right)+\left\|E_{u d}\right\|\right] \leq-\frac{1}{2} \lambda_{\min }\left(\Gamma^{4}\right) } \\
\cdot & \|\tilde{x}\|^{2}+\lambda_{\max }(\Omega)\left(\varsigma_{6}+\varsigma_{7}\right)\left\|\Gamma^{3}\right\|_{2}\|\tilde{x}\|^{2}+\lambda_{\max }(\Omega) \\
\cdot & E_{u d}^{*} \leq-\frac{1}{2} \lambda_{\min }\left(\Gamma^{4}\right)\|\tilde{x}\|^{2}+\lambda_{\max }(\Omega)\left(\varsigma_{6}+\varsigma_{7}\right) \\
\cdot & \lambda_{\max }\left(\Gamma^{3}\right)\|\tilde{x}\|^{2}+\lambda_{\max }(\Omega) E_{u d}^{*}
\end{aligned}
$$

$$
\begin{aligned}
& -\frac{1}{2} \lambda_{\min }\left(\Gamma^{4}\right)\|\tilde{x}\|^{2} \\
& +\lambda_{\max }(\Omega)\left(\varsigma_{6}+\varsigma_{7}\right) \lambda_{\max }\left(\Gamma^{3}\right)\|\tilde{x}\|^{2} \\
& +\lambda_{\max }(\Omega) E_{u d}^{*}
\end{aligned}
$$

Let

$$
\begin{aligned}
\beta^{*}= & \frac{1}{2} \lambda_{\min }\left(\Gamma^{4}\right)-\frac{\lambda_{\max }\left(J^{-1} \mathrm{k}\right) \lambda_{\max }\left(\Gamma^{2}\right)}{4} \\
& -\lambda_{\max }(\Omega)\left(\varsigma_{6}+\varsigma_{7}\right) \lambda_{\max }\left(\Gamma^{3}\right),
\end{aligned}
$$

and we have

$$
\begin{aligned}
\dot{V} \leq & -\sum_{i=1}^{4} \eta_{i}^{*}\left\|S_{i}\right\|^{2}-\sum_{i=1}^{3} \frac{1}{\alpha_{i+1}^{*}}\left\|r_{i+1}\right\|^{2}-\beta^{*}\|\tilde{x}\|^{2} \\
& +\lambda_{\max }(\Omega) E_{u d}^{*}+\sum_{i=2}^{3} \frac{\left\|\Xi_{i+1}\right\|^{2}}{4} \leq-2 \rho V+\Upsilon
\end{aligned}
$$

where

$$
\begin{aligned}
& \rho \in\left[0, \min \left\{\eta_{i}^{*}, \frac{1}{\alpha_{i}^{*}}, \frac{\beta^{*}}{\lambda_{\max }\left(\Lambda^{3} \Omega\right)}\right\}\right], \\
& \Upsilon=\sum_{i=2}^{3} \frac{\left\|\Xi_{i+1}\right\|^{2}}{4}+\lambda_{\max }(\Omega) E_{u d}^{*} .
\end{aligned}
$$

If we choose $\eta_{i}, \Gamma$, and $H$, and we let $\rho \geq \Upsilon / 2 \mu$, when $(t)=\mu$, $\dot{V} \leq 0$.Therefore, $V \leq \mu$ is invariant set and we have

$$
0 \leq V \leq \frac{\Upsilon}{2 \rho}+\frac{[V(0)-\Upsilon]}{\left(2 \rho e^{-2 \rho t}\right)}
$$

If we choose the value appropriately, we can make $\Upsilon / 2 \rho$ small enough; the tracking error and observation error converge to a small neighborhood of the origin; that is, the tracking error of the closed-loop system and the observation error are semiglobally consistent and ultimately bounded and can be arbitrarily small by choosing the appropriate parameters.

\section{Simulation}

To demonstrate the effectiveness of the proposed controller, we perform simulation studies with the model of flexible joint robot (1) and (2). We choose the parameters of the robot in [17]. The reference trajectories of the three joints are

$$
q_{l d}= \begin{cases}\frac{\pi t^{2}}{32} & (\mathrm{t} \leq 2 \mathrm{~s}) \\ \frac{\pi}{4}-\frac{\pi(\mathrm{t}-4)^{2}}{32} & (2 \mathrm{~s}<\mathrm{t} \leq 4 \mathrm{~s}) \\ \frac{\pi}{4} & (\mathrm{t}>4 \mathrm{~s})\end{cases}
$$

A simple PID controller has been developed in simulations for motor control for the three flexible joints. For simplicity, 'Ref' represents the reference trajectories; as shown in Figure 2, the position of three links connected by three flexible joints show an obvious vibration. Therefore, effective control must be taken to achieve vibration suppression.

Besides the proposed controller, we also perform the comparative studies using the conventional dynamic surface controller, and both controllers have the same parameters. It is assumed that the position and the velocity of link are measurable in the method using the conventional dynamic surface controller. In the following simulation studies, we assume the estimated mass parameters of link dynamic model deviate from the actual values, and the link dynamic model is subject to a bounded external disturbance. The bounded external disturbance is $\tau_{d l}=50 \sin (\pi / 1.25)$. The actual values of three links and payload are half of the nominal values in the simulation. In the following simulation, 'DSC' represents the trajectories using the conventional dynamic surface controller that requires link information, and 'ODSC' represents trajectories using the controller proposed in this paper that does not require link information. Results of simulation are shown in Figures 3-9. 

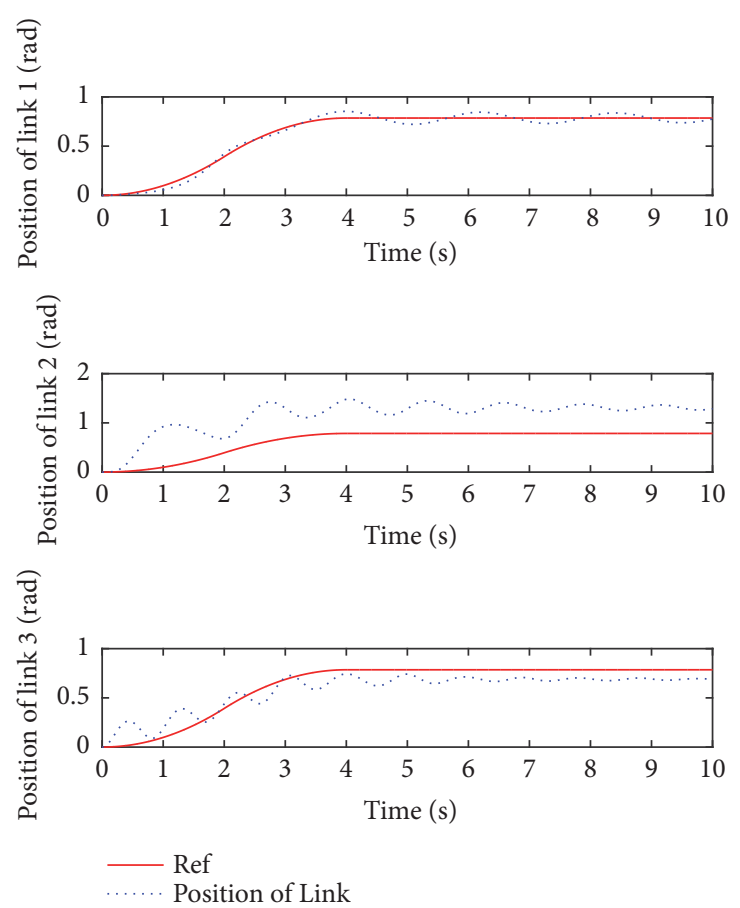

FIgURE 2: Response of three links.
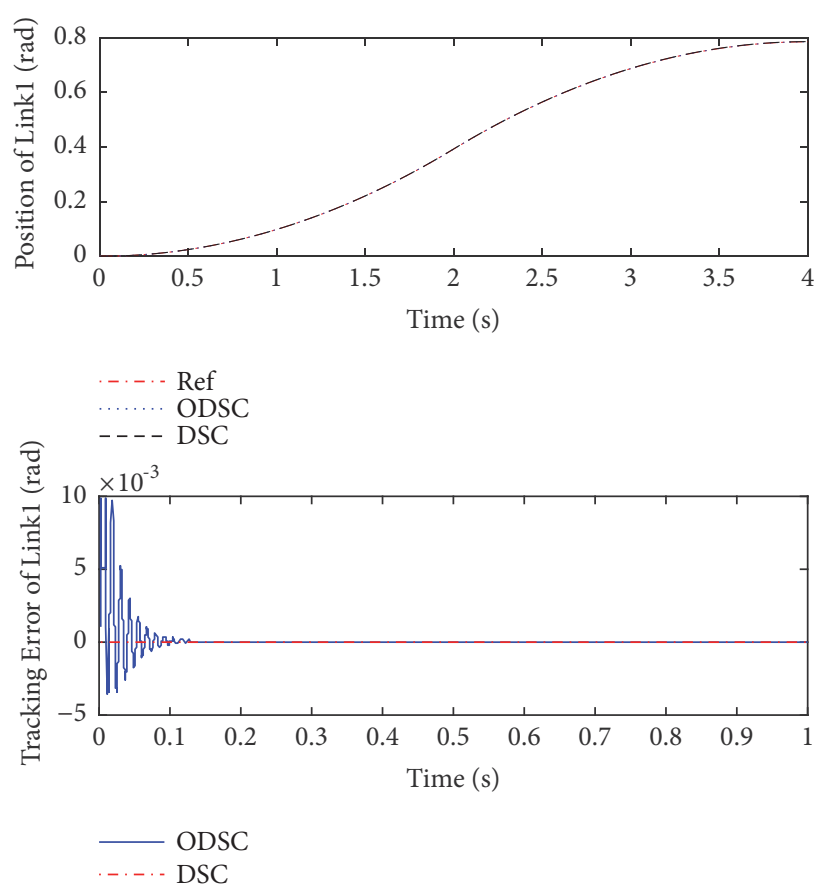

FIgURE 3: The position and tracking error of link1.

It can be observed from Figures 3, 4, and 5 that even if the estimated parameters have large deviations and the robot is subjected to large external disturbance, the state observer can still accurately estimate the positions and velocities of the links. The controller designed by using dynamic surface method based on state observer proposed in this paper can effectively achieve accurate trajectory tracking of link and suppress vibration of the flexible joints. Compared to the conventional dynamic surface controller using actual values, the control effect of the proposed controller is almost the same. The simulation results show that the proposed controller is robustness to external disturbance and the parameter uncertainty of the mass of the mass of the links and payload. Figure 6 shows the 

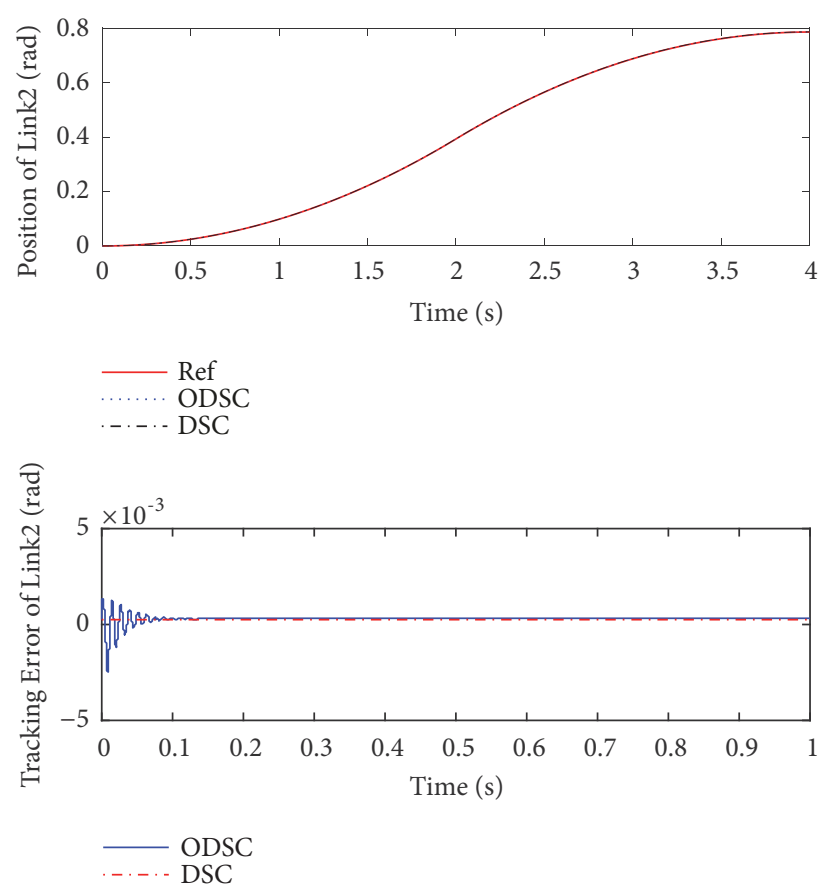

FIGURE 4: The position and tracking error of link2.
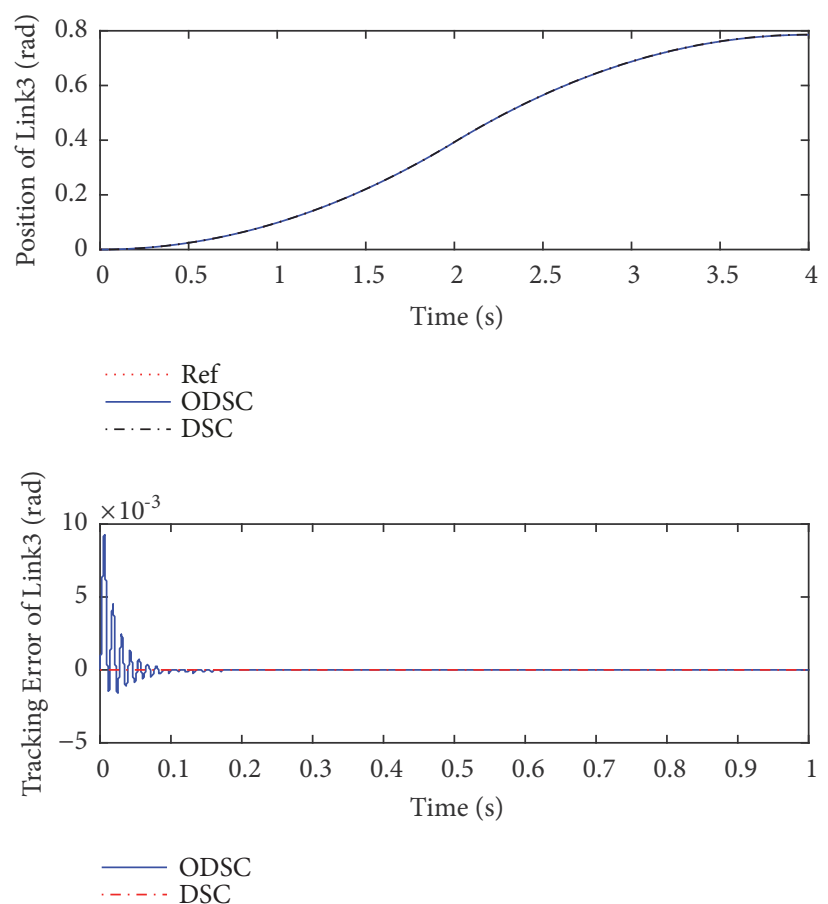

FigURE 5: The position and tracking error of link3.

control signals of three joints using the proposed controller.

Figures 3, 4, and 5 show that the controller proposed in this paper can achieve good trajectory tracking effect. Although the tracking error no longer converges to zero, it can converge to any small area associated with the design parameters, which is consistent with that the introduction of the first-order filter can still guarantee the uniform asymptotic stability of the whole system proved in Section 4.2. Figures 7, 8, and 9 show that the observer can approach the actual values with any small precision when the dynamic model has uncertain parameters, 

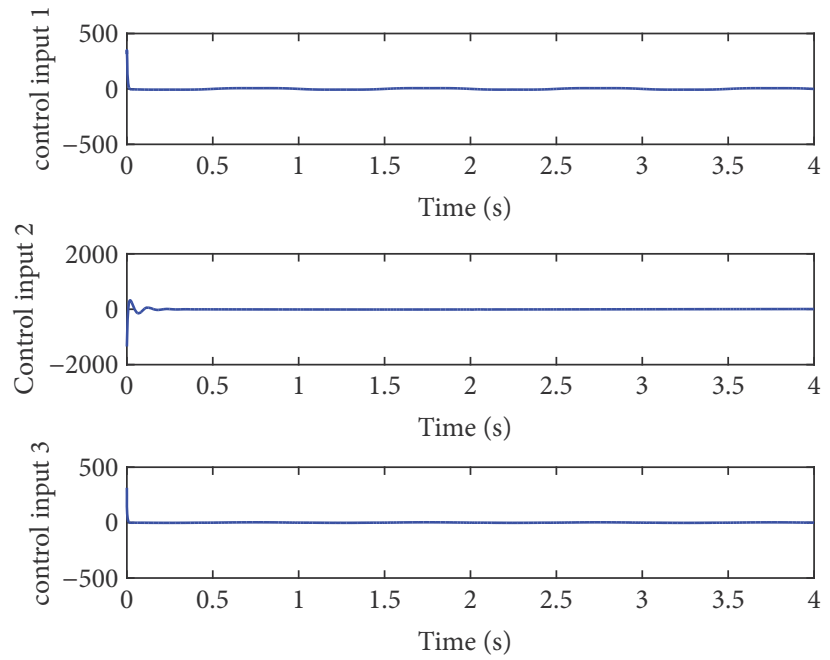

FIGURE 6: Control input of ODSC.
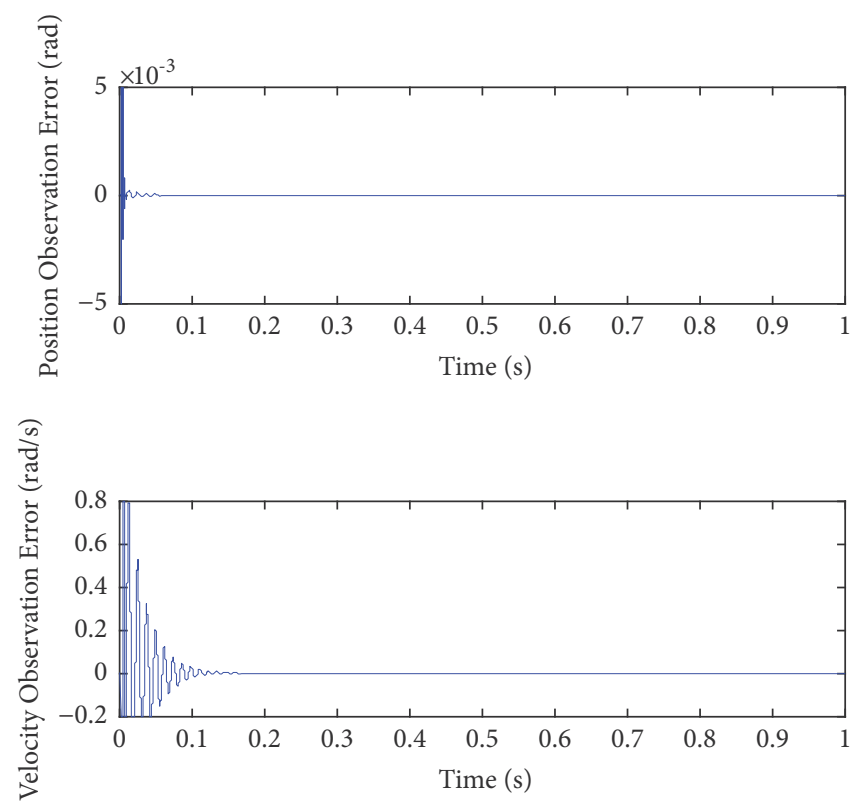

FIGURE 7: The estimation error of position and velocity of link1.

which means that the observer is robust to the uncertainty.

Figures 3, 4, and 5 show that the proposed controller achieves the same response as the conventional dynamic surface controller requiring the link information. Therefore, the proposed controller based on motor state feedback can still achieve the accurate trajectory tracking control for the flexible joint robot in the absence of the link state information. The proposed method does not need to modify the physical structure of existing industrial robot and will further facilitate its practical applications.

\section{Conclusions}

A controller for flexible joint robot with uncertain model is proposed based on motor state feedback. Considering the link state information cannot be obtained, an observer is designed to estimate the link state information. Considering the uncertain model and the external disturbance, a dynamic surface controller is proposed based on link state observer. The controller based on the observer compared to backstepping controller avoids the repeated differentiation problem. At the same time, the dynamic surface method avoids the measurement of high order signal. The Lyapunov stability proves that the controller based on observer makes the closed-loop system error signal consistent and ultimately bounded. The introduced first-order filter no longer makes the tracking error converge to zero, but can converge to an arbitrarily small area related to design parameters. The simulation results show that the designed controller has a good trajectory tracking effect, which effectively suppresses the residual vibration of the flexible joint robot. Moreover, 

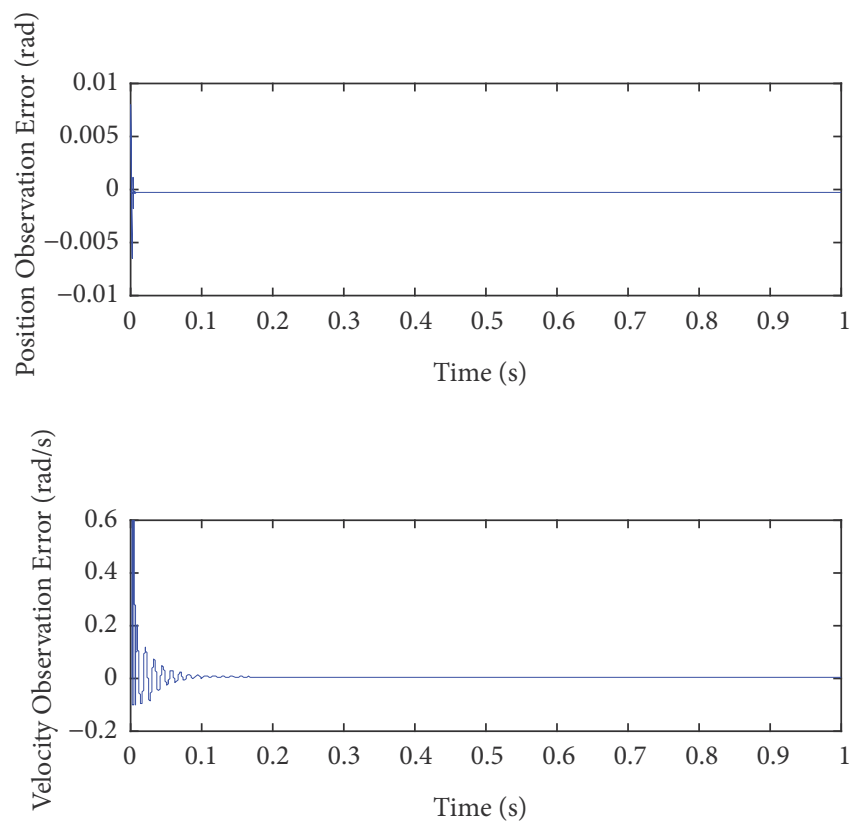

FIGURE 8: The estimation error of position and velocity of link2.
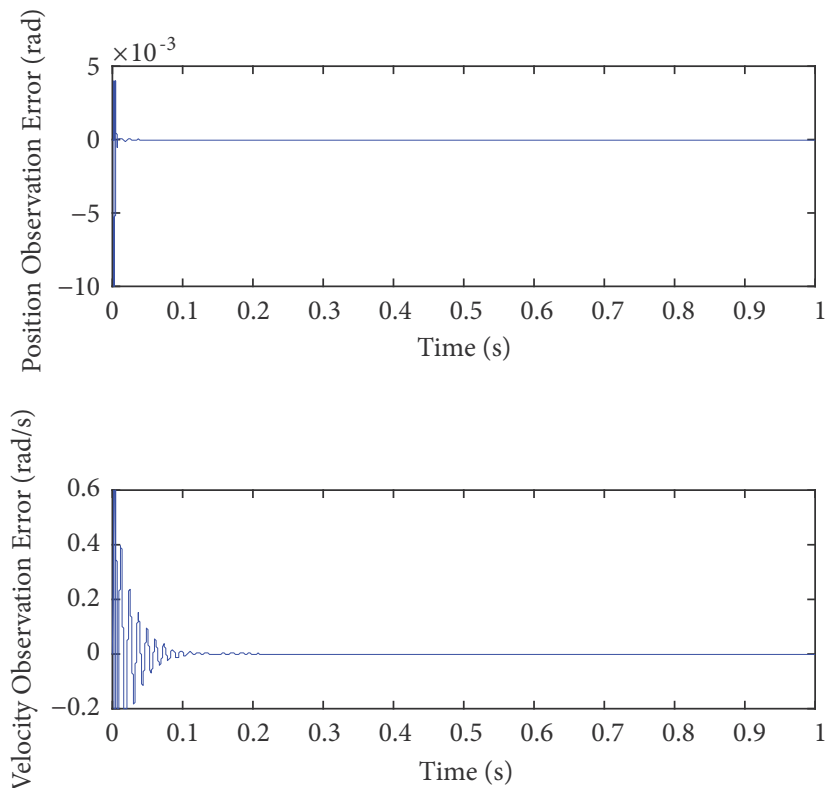

FIGURE 9: The estimation error of position and velocity of link3.

the proposed controller and observer are robust to the uncertainty and disturbance of the link dynamic. The proposed controller can be directly applied in the industrial application and do not need install additional sensors, which is very important for industrial applications.

\section{Data Availability}

The data used to support the findings of this study are available from the corresponding author upon request.

\section{Conflicts of Interest}

The author declares that there are no conflicts of interest regarding the publication of this paper.

\section{Acknowledgments}

This work is supported by the fundamental research funds for the central universities (no. 2015zcq-ly-02). 


\section{References}

[1] L. Wang, "Collaborative robot monitoring and control for enhanced sustainability," The International Journal of Advanced Manufacturing Technology, vol. 81, no. 9-12, pp. 1433-1445, 2015.

[2] X. Liu, C. Yang, Z. Chen, M. Wang, and C. Su, "Neuro-adaptive observer based control of flexible joint robot," Neurocomputing, vol. 275, pp. 73-82, 2018.

[3] International Organization for Standardization (ISO), "Robots and robotic devices-Collaborative robots [OL]," 2017, https:// www.iso.org/standard/62996.html.

[4] J. Choi, S. Hong, W. Lee, S. Kang, and M. Kim, "A robot joint with variable stiffness using leaf springs," IEEE Transactions on Robotics, vol. 27, no. 2, pp. 229-238, 2011.

[5] D. Navarro-Alarcon, Z. Wang, H. M. Yip, Y.-H. Liu, P. Li, and W. Lin, "A method to regulate the torque of flexiblejoint manipulators with velocity control inputs," in Proceedings of the 2014 IEEE International Conference on Robotics and Biomimetics, IEEE ROBIO 2014, December 2014.

[6] W. He, Y. Chen, and Z. Yin, "Adaptive neural network control of an uncertain robot with full-state constraints," IEEE Transactions on Cybernetics, vol. 46, no. 3, pp. 620-629, 2016.

[7] M. Chen and G. Tao, "Adaptive fault-tolerant control of uncertain nonlinear large-scale systems with unknown dead zone," IEEE Transactions on Cybernetics, vol. 46, no. 8, pp. 1851-1862, 2016.

[8] M. Chen and S. Ge, "Adaptive neural output feedback control of uncertain nonlinear systems with unknown hysteresis using disturbance observer," IEEE Transactions on Industrial Electronics, vol. 62, no. 12, pp. 7706-7716, 2015.

[9] M. Chen, P. Shi, and C.-C. Lim, "Robust constrained control for MIMO nonlinear systems based on disturbance observer," Institute of Electrical and Electronics Engineers Transactions on Automatic Control, vol. 60, no. 12, pp. 3281-3286, 2015.

[10] W. He, Y. Ouyang, and J. Hong, "Vibration control of a flexible robotic manipulator in the presence of input deadzone," IEEE Transactions on Industrial Informatics, vol. 13, no. 1, pp. 48-59, 2017.

[11] W. He and S. Zhang, "Control design for nonlinear flexible wings of a robotic aircraft," IEEE Transactions on Control Systems Technology, vol. 25, no. 1, pp. 351-357, 2017.

[12] D. Swaroop, J. K. Hedrick, P. P. Yip et al., "Dynamic surface control for a class of nonlinear systems," IEEE Transactions on Automatic Control, vol. 45, no. 10, pp. 1893-1899, 2000.

[13] Z. Peng, D. Wang, and J. Wang, "Predictor-based neural dynamic surface control for uncertain nonlinear systems in strict-feedback form," IEEE Transactions on Neural Networks and Learning Systems, vol. 28, no. 9, pp. 2156-2167, 2017.

[14] D. Wang and J. Huang, "Neural network-based adaptive dynamic surface control for a class of uncertain nonlinear systems in strict-feedback form," IEEE Transactions on Neural Networks and Learning Systems, vol. 16, no. 1, pp. 195-202, 2005.

[15] S. J. Yoo, J. B. Park, and Y. H. Choi, "Adaptive dynamic surface control of flexible-joint robots using self-recurrent wavelet neural networks," IEEE Transactions on Systems, Man, and Cybernetics, Part B: Cybernetics, vol. 36, no. 6, pp. 1342-1355, 2006.

[16] J. Kim and E. A. Croft, "Preshaping input trajectories of industrial robots for vibration suppression," Robotics and ComputerIntegrated Manufacturing, vol. 54, pp. 35-44, 2018.

[17] F. Abdollahi, H. A. Talebi, and R. V. Patel, "A stable neural network-based observer with application to flexible-joint manipulators," IEEE Transactions on Neural Networks and Learning Systems, vol. 17, no. 1, pp. 118-129, 2006.

[18] Y. H. Qiang, F. S. Jing, Z. G. Hou et al., "Dynamic modeling and vibration mode analysis for an industrial robot with rigid links and flexible joints," in Proceedings of the 2012 24th Chinese Control and Decision Conference, CCDC 2012, pp. 3317-3321, China, May 2012.

[19] M. Jankovic, "Observer based control for elastic joint robots," IEEE Transactions on Robotics and Automation, vol. 11, no. 4, pp. 618-623, 1995. 


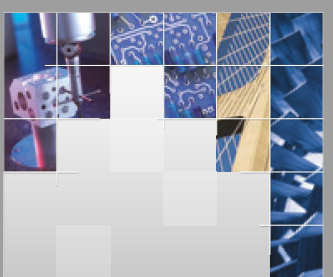

\section{Enfincering}
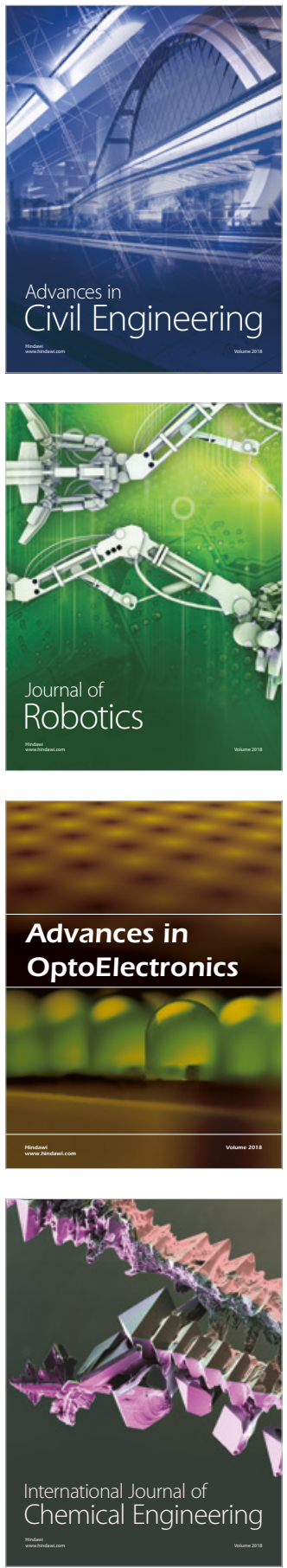

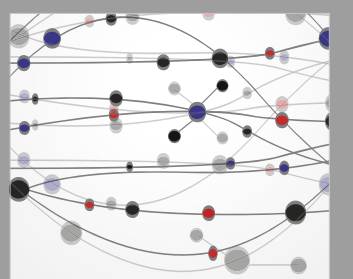

\section{Rotating \\ Machinery}

The Scientific World Journal

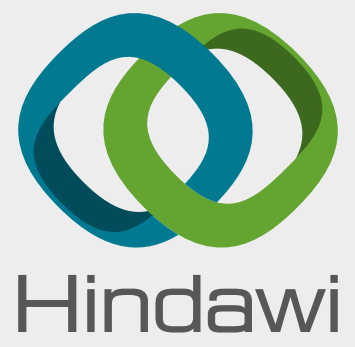

Submit your manuscripts at

www.hindawi.com
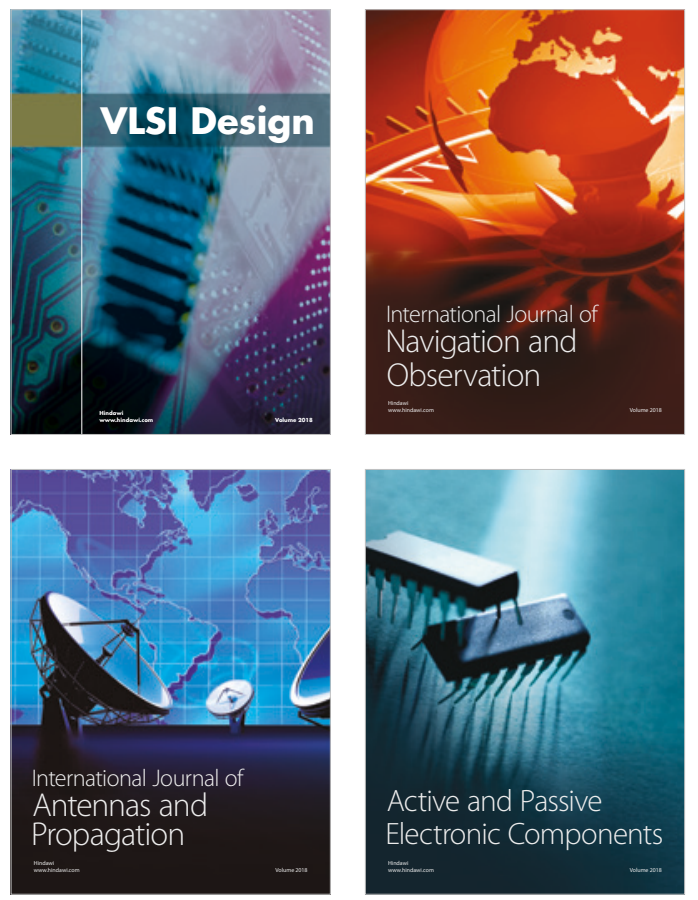
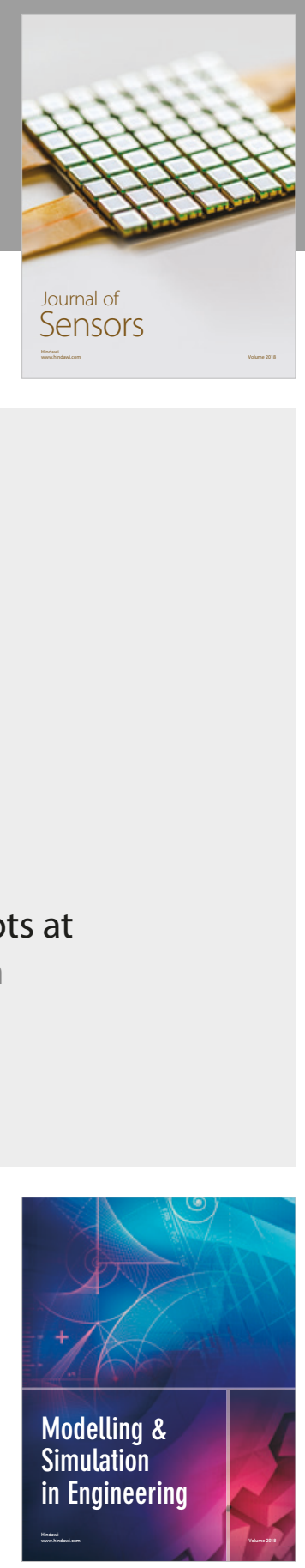

\section{Advances \\ Multimedia}
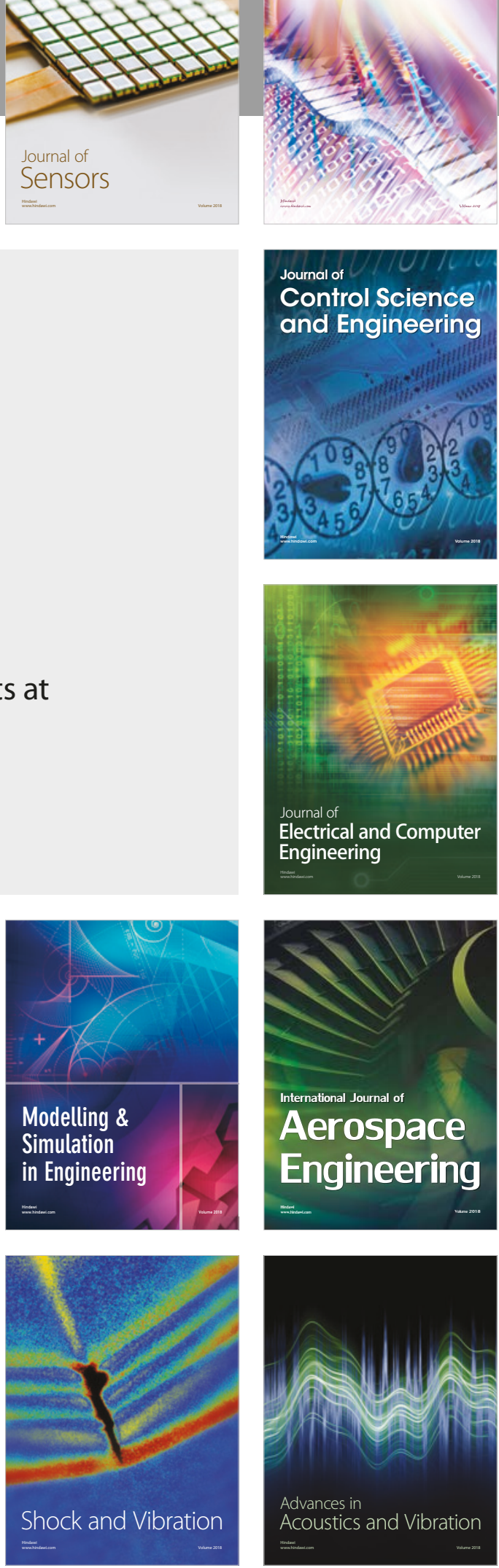